\title{
Differential interferometry in heat transfer
}

\author{
V RAMMOHAN RAO, C B SOBHAN* and S P VENKATESHAN \\ Department of Mechanical Engineering, Indian Institute of Technology, \\ Madras 600036, India \\ * Present Address: Department of Mechanical Engineering, Regional \\ Engineering College, Calicut 673601, India
}

MS received 13 September 1990

\begin{abstract}
In this paper the optical methods in heat transfer applications are described briefly. In particular, interferometric methods are discussed in detail as applied to the study of natural convection heat transfer. Of the most popular interferometers, viz. the Mach Zehnder Interferometer (MZI) and the Differential Interferometer (DI), the DI is considered in detail. The theoretical and constructional features of a DI are outlined. The procedures of heat transfer measurement and the temperature profile estimation are explained. The advantages and limitations of the two interferometers are compared with the help of a sample calculation. The experimental analyses of three cases, viz. (1) isothermal vertical flat plate, (2) single vertical fin attached to a heated horizontal base, and (3) horizontal fin array, are described. Typical heat transfer and temperature profile results are presented. Using the estimated temperature profiles isotherms are plotted. It is possible to get fairly good heat transfer and temperature profiles using a DI. The instrument is specially useful in applications involving short optical path lengths because of its superior resolution as compared to MZI.
\end{abstract}

Keywords. Differential interferometry; heat transfer; temperature profile estimation.

\section{Introduction}

Optical methods are a powerful means of measurement in many fields of science and engineering, such as stress analysis, fluid mechanics and heat transfer. These methods are based on specific properties of light such as reflection, diffraction, polarization and interference. Optical methods are very convenient to use due to the various advantages over other methods of measurement, and in most situations, whether steady state or transient, yield accurate qualitative and quantitative results. 


\section{Optical methods in the measurement of heat transfer}

Use of optical methods in the measurement of heat transfer is superior to other methods such as calorimetric and probe techniques. This is because they are generally non-intrusive in nature and hence do not disturb the flow field. The most widely used optical technique in the measurement of heat transfer falls under the category referred to as the Index of Refraction Method. In this method, the density and temperature fields associated with a flow region are calculated, using a measurement of the refractive index or its spatial derivatives for light of a given wavelength, in the medium. When there are temperature non-uniformities occurring in the medium, and there is an associated density field, the refractive index of the medium will be different at different locations in the medium, being a function of the density. This change in the refractive index causes a corresponding deformation in the wave front of a light beam when it passes through the medium. If the initial shape of the wave front is known in the unheated condition (that is, with a uniform temperature field), the amount of deformation in the wave front can be used as an indication of temperature non-uniformities in the medium. The optical methods used for measurement of heat transfer using the index of refraction method employ this principle.

The deformed wave front as explained above is measured generally using any one of the following methods:

(a) shadowgraph and schlieren methods utilizing the deflection of light in the medium;

(b) interferometric methods using the optical path differences in the medium, and the phenomenon of interference of light.

Only interferometric methods are considered in this paper.

\section{Interferometric methods}

Interferometric methods are widely used for quantitative measurement of temperature or heat flux fields. These methods utilize the interference of coherent light beams, in which additional phase lags are introduced when they pass through a density field. In the Mach-Zehnder Interferometer (MZI), one of the coherent beams passes through the test field; and the other passes through an isothermal field and is used as a reference beam. On recombination, the two beams form a fringe pattern which contains information about the temperature distribution. In the Differential Interferometer (DI) the two beams pass through different locations within the test field, separated by a small but known distance. While recombining, these produce a fringe pattern which carries information about the difference of the temperatures between these two locations, or in other words, the spatial derivative of temperature in the field.

\section{Theoretical basis for the index of refraction methods}

The relationship between the density of a homogeneous medium and its refractive index for light is given by the Lorentz-Lorenz relation

$$
\left(n^{2}-1\right) /\left[\rho\left(n^{2}+2\right)\right]=C .
$$


Equation (1) when applied to a gas with $n \approx 1$ reduces to the following:

$$
(n-1) / \rho=1 \cdot 5 C,
$$

where, $C$ is the Gladstone-Dale constant, characteristic of the gaseous medium, and independent of the pressure of the gaseous medium (Edser 1949). For air, $C=0.1506 \times$ $10^{-3} \mathrm{~m}^{3} / \mathrm{kg}$.

Substituting $(P / R T)$ in place of $\rho$, for an ideal gas as the medium, (2) yields (assuming $P=$ constant)

$$
\partial n / \partial y=-1 \cdot 5\left(C P / R T^{2}\right)(\partial T / \partial y) .
$$

In the interferometric method, the shift in the interference fringes at any location can be shown to be proportional to the temperature (in the MZI) or its first derivative (in the DI) according to the type of the instrument used. These are treated in greater detail below.

\section{Interferometry in heat transfer measurement}

The interferometric methods utilize the fact that when an additional phase shift is introduced between two coherent beams of light interfering and forming fringes, there is a deformation in the fringe pattern. This deformation is used as a measure of the temperature or the heat flux as the case may be. Various methods are employed in interferometers for obtaining the coherent beams, as these have to be produced from a single source of light using some optical system. The generally used methods are listed below (Hauf \& Grigull 1970).

(1) Division of amplitude;

(2) division by diffraction gratings;

(3) birefringent splitting of polarized light.

The most common types of interferometers used for heat transfer measurement are the Mach-Zehnder interferometer (Dyson 1970; Dosanjh 1971) and the differential interferometer (Black \& Carr 1971; Black \& Norris 1974).

In the MZI, a splitter plate is used to divide a light beam and to obtain two coherent light beams. One of these travels through the test section and the other is kept as a reference beam. When these combine together, they produce interference fringes. Figure 1 shows the working principle of the MZI. The interference fringes formed are deformed if there is a temperature field associated with the test section. Isotherms are visualized from the fringe patterns. The local heat flux values, if needed, are calculated by performing numerical differentiation. Hence this type of interferometer is preferred when the temperature field is to be mapped. However, the MZI has some inherent disadvantages as listed below.

(1) The instrument is very sensitive to any misalignment of the components, as the spatial distance between the two beams is very large.

(2) Setting up of the instrument is time-consuming.

(3) Calculation of the heat transfer coefficients requires the temperature gradients, which cannot be directly obtained in the classical MZI. With the modified forms, it 
(a)
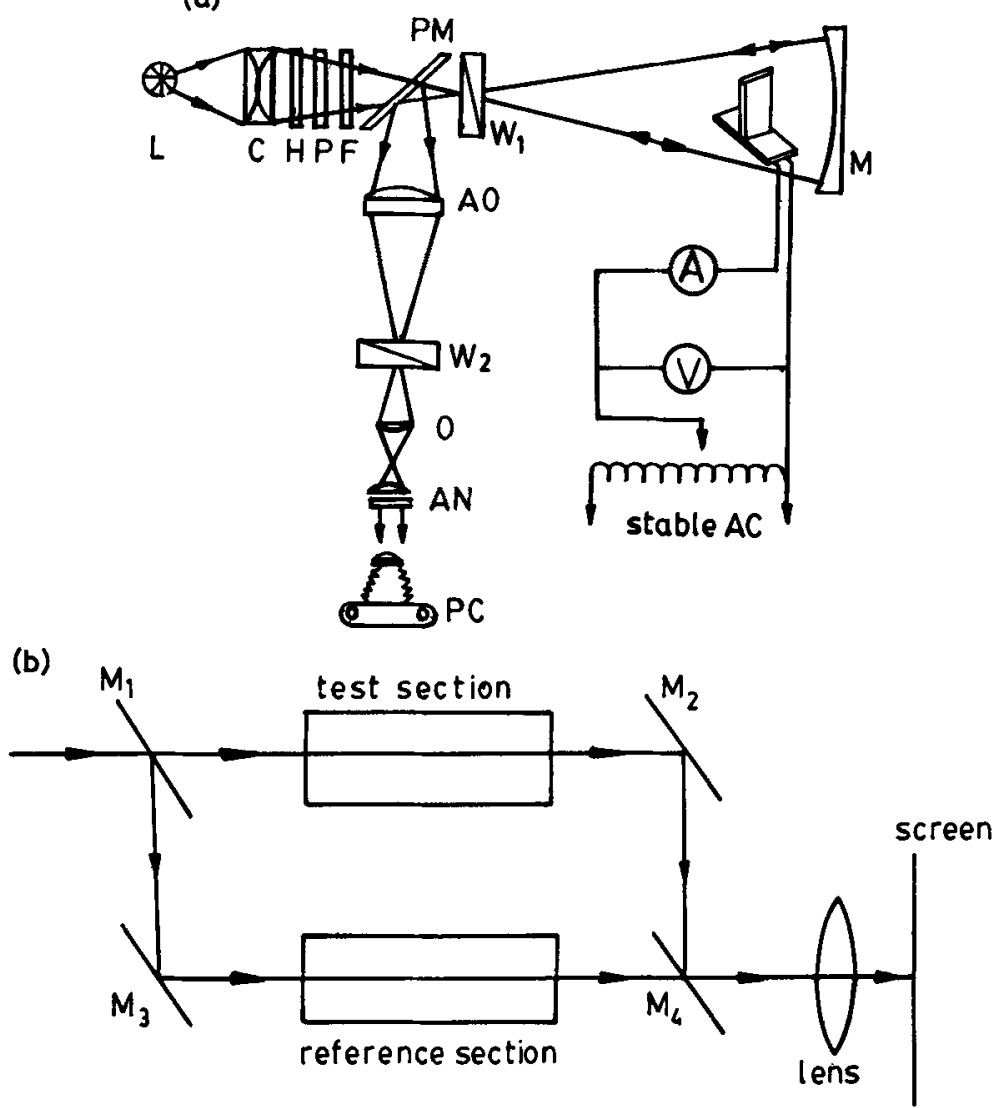

Figure 1. (a) Schematic diagram showing the working principle of the differential interferometer. L-light source; $\mathbf{C}$-condenser; $\mathbf{H}$ - heat filter; $\mathbf{F}$-colour filter; $\mathbf{P}$-polariser; PM-partial mirror. $\mathbf{W}$-Wollaston prism; $\mathrm{M}$-mirror; $\mathbf{A O}$-achromatic objective; $\mathrm{O}$-ocular; AN-analyser; A-ammeter; V-voltmeter; PC-photographic camera. (b) Schematic diagram of the Mach-Zehnder interferometer (from Dyson 1970). The diagram shows a reference beam and a test beam and a recombination of these beams to yield an interference pattern.

is possible, but it requires complicated and time-consuming movements of the mirror and splitter plates (Hauf \& Grigull 1970). These conjugate movements may introduce errors, and also make the instrument more sensitive to misalignment.

In the DI, beam-splitting is done by a birefringent prism, which produces light rays that travel close to each other. There is no reference beam as such, but both the rays pass through the test section at locations adjacent to each other. This introduces phase lag in both the rays which are proportional to the density values at the two locations. On recombination these give rise to fringes which are deformed, and the shift in the fringes carries information about the difference in densities of the medium at these two locations. If the spatial separation between the two points under consideration is known, the fringe shift can be used to find the derivatives of the temperature in the field, which yields the local conductive heat flux values, integrated along the path of light. The theoretical aspects and the constructional details of the differential interferometer are given in greater detail in the sections to follow. 


\section{The differential interferometer}

Differential interferometers are used for observation and measurement of various phenomena, some of which are listed below.

(1) Fluid flow in wind tunnels.

(2) Density and concentration fields associated with diffusion in liquids and gases.

(3) Property variations within transparent plastics and glasses.

(4) Heat flux and temperature field patterns associated with convective heat transfer in liquids and gases.

As the differential interferometer does not utilize any slit or stop for beam-splitting, the light rays are of high intensity and photographing of quickly varying processes can be effectively done with short exposure times (Schrader 1978).

The differential interferometer has the following advantages when compared with other techniques used in heat transfer measurements.

(1) It is non-intrusive, being an optical technique.

(2) The DI is superior to other interferometric methods in heat transfer studies since heat fluxes can be directly measured.

(3) Complete information about the local temperature gradients in the flow field can be recorded in a single photograph.

(4) Instrumentation is much simpler than that with calorimetric methods because elaborate procedures for estimating heat losses or for eliminating heat losses from the set-up are not necessary, as the differential interferometer does not rely on heat balance to obtain the heat fluxes.

(5) Adjustments of the instrument are fairly quick and easy.

\subsection{Constructional details of the DI}

When light plane-polarized in a particular direction, passes through certain crystals, the ordinary and extraordinary components travel with different velocities. In other words, the crystal has different refractive indices for the two components. If a prism is made of such a material (e.g. quartz, calcite), each light ray while emerging produces two rays which are separated from each other in space as well as in phase. These can be used as the interfering light rays. This method is utilized in producing coherent light rays using Wollaston prisms. The Wollaston prism (Black \& Norris 1974; Smith \& Thomson 1977) is made by cementing together two wedges of a birefringent material. The two wedges are of the same dimensions but their optic axes are perpendicular to each other as shown in figure 2 . The ordinary and extraordinary components are refracted differently. The ordinary component behaves normally but the other component does not follow Snell's law of refraction. The two wave fronts touch each other only along the optic axis of the crystal. The refraction at the crystal face depends on the direction of incidence, on the polarization and the orientation of the optic axis in the crystal. Light plane-polarized at $45^{\circ}$ to the principal direction first enters the crystal whose optic axis is perpendicular to the refracting surface. In this case, the rays and the wave normals coincide. This part of the prism makes sure that the total physical length of travel of all the rays in the material is the same. Rays from the first crystal fall on the second one whose optic axis is parallel to the refracting surface. Within this crystal, the ordinary and extraordinary rays travel with different velocities. 


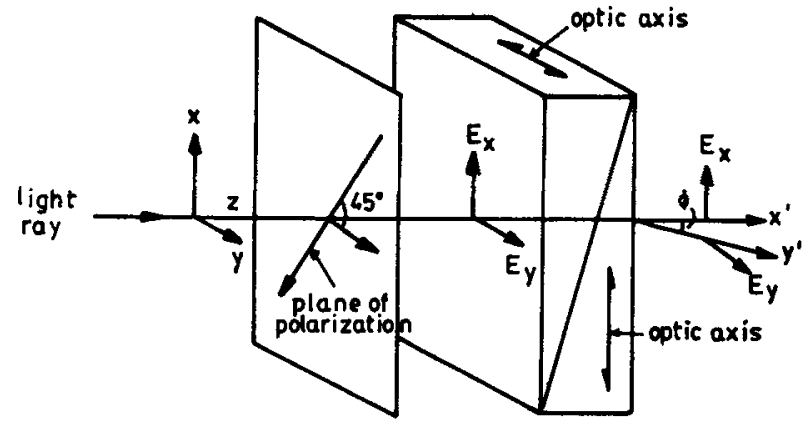

Figure 2. Birefringent splitting of a light ray using a Wollaston prism (from Black \& Carr 1971). $x$ direction of propagation of ordinary ray; $\boldsymbol{y}$-direction of propagation of extraordinary ray; $\phi$-angular separation between the two rays, in the plane normal to the paper.

When the rays emerge from the second crystal, the phase relation between the two components, which are polarized parallel to and perpendicular to the optic axis, changes by an amount proportional to the thickness of the wedge at the locations where light passes through it.

The two rays while emerging have a difference in phase angle as given by the following relation (Smith \& Thomson 1977)

$$
\phi=(2 \pi / \lambda)\left(n_{0}-n_{e}\right) w .
$$

These rays are also separated in space depending on the wedge angle of the prism.

Figure 1 also shows the schematic diagram of the DI used in the present experimental work. The light from the light source $\mathrm{L}$ is projected by the condensing lens $C$ on to the Wollaston prism $W_{1}$, through a heat filter $H$, polarizer $P$ and $a$ colour filter $F$ and through the partial mirror PM. The polarizer has the axis of polarization oriented at an angle of $45^{\circ}$ with respect to the $x-z$ plane as shown in figure 2. Hence the light that enters the prism $W_{1}$ is polarized in that direction. The colour filter is used for obtaining a monochromatic light beam. The Wollaston prism is kept at the centre of curvature of the concave mirror $M$. The light which arrives at the mirror is reflected on itself. At the partial mirror PM, it is deflected side ways through the telescope.

The telescope consists of the achromatic objective AO, a second Wollaston prism $\mathrm{W}_{2}$, ocular $\mathrm{O}$ and analyser $\mathrm{AN}$. The photographic camera $\mathrm{PC}$ can be attached to the eye piece of the telescope for taking photographs.

Interference fringes are seen if the Wollaston prism $\mathrm{W}_{2}$ is introduced in the telescopic part, and this is used for quantitative measurement. If this prism is removed, what is known as the no-fringe or infinite fringe spacing pattern is obtained, where the fringes are absent, but contrasts corresponding to the different regions of temperature gradients are seen. This may be used for qualitative observation of the heat transfer field. Analyser AN is similar to the polarizer in construction. It is the analyser which brings together the light rays so that an interference fringe pattern is obtained. Hence, with the telescopic section, all the optical phenomena taking place in the forward motion of light are reversed except those which take place when light travels through the test section. On return through the test section. the phase lag in the light is only doubled. Thus the information regarding the test section is conserved during the process.

\subsection{Physical principle of the DI}

It has been explained in $\$ 6.1$, how two coherent rays of light, corresponding to each parent ray, emerge from the Wollaston prism. These rays which are slightly divergent 


\section{- - unheated heated}

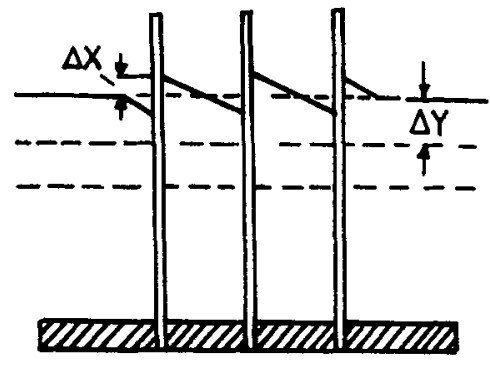

Figure 3. Schematic diagram of the heated fin-base assembly showing the deflection in the otherwise parallel fringes due to a temperature field in the test section. The fringe shifts indicate the gradient of temperature normal to the fin flat.

pass through the test section with a separation of $\Delta y$ with each other, which for yellow light is given by (Schrader 1978)

$$
\Delta y=0.0182 d \tan \delta
$$

If there is a heat transfer in the test section, such as the one adjacent to a heated object, the two rays pass through regions of different temperature and densities. Hence there exists an optical path difference (OPD) between the two electric vector components given by

$$
\mathrm{OPD}=\int n \mathrm{~d} z .
$$

Assuming that the refractive index $n$ is constant along the direction of light travel, from (5) (Black \& Carr 1971)

$$
\mathrm{OPD}=L \Delta n=m \lambda
$$

It is this difference in the optical path which causes the shift in the interference fringes. The fringe patterns obtained by the introduction of prism $W_{2}$ are separated by a distance $\Delta Y$ from each other. When the additional phage lag comes in from the heated test section, there is a deflection in the otherwise parallel fringes, as shown in figure 3. This deflection is measured in terms of a fringe shift factor $m$ which is the ratio of the amount of deflection $\Delta X$ to the fringe width $\Delta Y$ as shown in figure 3 . The fringes are straight, far away from the surface and are displaced only near the heat transfer surface as shown and the temperature gradient is assumed to be small.

The gradient of refractive index can then be approximated as

$$
\partial n / \partial y \approx \Delta n / \Delta y
$$

It is thus seen that the above is not a good representation for steeply varying temperature fields like those that occur in the presence of shock waves etc.

As both the ordinary and extraordinary components of the light ray undergo refraction in a double refracting prism, (5) for $\Delta y$ is modified as

$$
\Delta y=(2 d)(0.0182) \tan \delta
$$

Combining (7), (8) and (9),

$$
\partial n / \partial y=\lambda m /(2 L d 0 \cdot 0182 \tan \delta) \text {. }
$$

Thus the gradient of the refractive index is obtained if the fringe shift factor $m^{\prime}$ is measured. 
If the prism $\mathrm{W}_{2}$ and the polarizer in figure 1 are turned, the measuring rays can be made to intersect the test section at any desired angle. If the analyser is turned by the same angle the interference fringes formed also have the same orientation. This makes the differential interferometer useful in measuring the heat flux values normal to surfaces oriented in any arbitrary direction.

\subsection{Measurement of heat transfer by the DI}

At a solid wall where there is convective heat transfer occurring to the ambient, the heat convected can be equated to the conduction through the fluid layer adjacent to the surface. That is,

$$
q_{w}=h\left(T_{w}-T_{\infty}\right)=-k_{w}(\partial T / \partial y)_{w} .
$$

Combining (3) with (10) one gets,

$$
-(\partial T / \partial y)=(2 / 3)\left\{\left(R T^{2} / C P\right) \lambda m /[2 L d 0.01182 \tan (\delta)]\right\} .
$$

Then (11) and (12) give,

and

$$
q_{w}=(2 / 3)\left\{\left(R T_{w}^{2} / C P\right) \lambda m /[2 L d 0.0182 \tan (\delta)]\right\} k_{w},
$$

$$
h_{w}=k_{w} R T_{w}^{2} \lambda m /\left[3 C P L d 0.0182 \tan (\delta)\left(T_{w}-T_{\infty}\right)\right] \text {. }
$$

Thus by the measurement of the fringe shift at the wall and the wall temperature, the heat transfer coefficient at the wall may be calculated.

\subsection{Obtaining the temperature profile}

As shown above by (12) the differential equation connecting the fringe shift and the temperature is of the form

$$
(\partial T / \partial y)+k^{\prime} m(y) T^{2}=0,
$$

where $k^{\prime}$ is a constant given by $(2 R \lambda) /(3 C P 2 L d 0.0182 \tan \delta)$.

This is a first order non-linear differential equation in which $k^{\prime}$ includes the DI and other constants and $m$ is the fringe shift factor. To solve this equation for the temperature exactly, one needs one boundary condition for temperature and the fringe shift distribution in the direction $y$. In general, it is observed that the surface temperature and the ambient temperature can be measured easily, which means that one has two boundary conditions to be satisfied by the solution of the above equation. If the measurements were accurate, using one boundary condition (or an initial condition) one would get the other boundary condition as a solution. As the measurements are always subject to error this is not a possible situation. An alternate choice would be a solution by statistical means. This can be done by approximating the temperature by a polynomial and the constants in the polynomial being obtained using the least squares principle. In other words, the solution satisfies the measured values with a least squares integrated residual. Details of the calculation procedure and the resulting errors are presented elsewhere (Rammohan Rao \& Venkateshan 1990). A few typical results will be presented later. 


\section{Comparison of MZI with DI}

In this section the working of an MZI will be discussed as compared to that of a DI. It is normally believed that the measurements using an MZI are more accurate than those using a DI (Hauf \& Grigull 1970). But, it is shown in the following that this is not so in general. In many situations the MZI yields poor results and its applicability is also limited as compared to a DI.

\subsection{Governing equation for an $M Z I$}

The relationship between refractive index $n$ and the density $\rho$ for air is given by the Lorentz-Lorenz relation (2) which may be recast in the form

$$
\left(n_{\infty}-1\right) /(n-1)=\left(\rho_{\infty} / \rho\right)=\left(T / T_{\infty}\right) .
$$

The ideal interferometry equation is (Hauf \& Grigull 1970)

$$
n_{\infty}-n=S \lambda / L
$$

from which one gets

where

$$
\left(T / T_{\infty}\right)=1 /(1-a S)
$$

$$
a=\left(2 \lambda R T_{\infty}\right) /\left(3 L C P_{\infty}\right)=2 \lambda / 3\left(C L \rho_{\infty}\right) \text {. }
$$

It is seen from the above that the number of fringes in a given temperature interval (say between the surface and the ambient) is larger the smaller the value of $a$. Hence the temperature resolution of the MZI depends inversely on the product $C L \rho_{\infty}$. For example, with water as the medium one can expect a much better resolution with a given path length than in air because of a density reduction in the latter by a factor of approximately $10^{3}$.

\subsection{Comparison of $\mathrm{DI}$ with $\mathrm{MZI}$}

In the DI the number of fringes per unit height of the object is independent of the dimensions of the object. In fact fringe spacing can be varied by selecting different Wollaston prisms near the eye piece. Another adjustment that is possible in the DI is that the fringes can be positioned anywhere along the height of the object. That means fringe spacing is not a limitation in DI, provided two or more interferograms are analysed for the same experimental run. Hauf \& Grigull (1970) mention that the measurements using MZI are more accurate than that using DI because DI gives a smaller number of interference lines. From the earlier discussion it is seen that this is not a serious limitation. In MZI the number of interference lines is a function of the temperature difference and the length of the test medium besides the operating variables (18). For example, for a particular temperature difference and other conditions there exists a minimum length of the test medium (object length) to get the desired number of interference lines. It will be shown in a later section that this is a serious constraint as far as MZI is concerned.

In the case of a DI the heat transfer is calculated directly as it gives the temperature gradient rather than the temperature itself. MZI gives the temperature difference as 
compared to a reference temperature. The heat transfer from the surface is calculated by a finite difference approximation using two or more temperatures near the surface. The calculated heat transfer values are accurate provided the distance between the two neighbouring isotherms is small. This means the accuracy of the calculated heat flux is independent of the resolution in the case of a DI. But for an MZI the resolution depends on various parameters like the test object length, the temperature difference etc.. Hence the errors involved are dependent on the test set-up geometry, the temperatures involved, the medium etc.. This is demonstrated by a sample calculation as below.

\subsection{Sample calculation}

Consider an isothermal vertical flat plate (described in $\$ 11.1$ below). The operating conditions and the geometry are as follows.

$$
\begin{aligned}
& T_{w}=341.8 \mathrm{~K}, T_{\infty}=301 \mathrm{~K}, P_{\infty}=101325 \mathrm{~Pa}, \\
& \text { height of the plate }=100 \mathrm{~mm}, \\
& \text { wavelength of the light used } \lambda=0.5461 \times 10^{-6} \mathrm{~m} \text { (yellow), } \\
& \text { length of the plate } L=60 \mathrm{~mm} \text {, } \\
& \text { Gladstone-Dale constant for air } C=0.1506 \times 10^{-3} \mathrm{~m}^{3} / \mathrm{kg} \text {. }
\end{aligned}
$$

With a DI the heat transfer calculations are straightforward using the expression $q_{x}=$ constant $\cdot m_{w} \cdot T_{w}^{2}$ which is a linear function of the fringe shift factor as $T$ is maintained constant. The fringe spacing used was $3 \mathrm{~mm}$ which means local measurements were possible with a minimum space interval of $1.5 \mathrm{~mm}$. If two interferograms were to be analysed for the same conditions with different DI settings, this interval could be halved. These heat transfer values agree very well with the available integral solution. The temperature profiles were also worked out at various locations from the leading edge (i.e. for various $G r_{x}$ ) using the method discussed in $\$ 6.4$ and the comparison was found to be excellent with the Ostrach's similarity solution. Now let us see what would be the situation if MZI is used for the same experiment.

The fringe number $S$ corresponding to the wall temperature (an isotherm) taking the fringe number for the ambient as zero can be determined from (18). For the experimental conditions given above, $a=0.03451$ from which we get $S=3$ near the wall. This means the MZI would give 4 interference lines corresponding to the ambient, $311 \cdot 7,323.2$ and 335.6 respectively. From this one can see how crude the heat transfer values evaluated from these temperatures would be. If the test set-up is 10 times longer i.e. $L=500 \mathrm{~mm}$, one would get about 21 fringes. To increase the number of interference lines i.e. to make the resolution better, it is necessary to change the values of one or more parameters in (18). However, if one does not have any control over the experimental conditions the MZI may not be useful at all. Having discussed the general features of the interferometric methods we present below details of the DI-based measurements that were performed in our laboratory.

\section{Components of the experimental set-up}

The experimental set up consists of the following components:

(1) differential interferometer; 
(2) test specimen;

(3) power input system;

(4) temperature measurement system;

(5) photographic camera.

Developed photographs are projected using a Pradovit slide projector and the measured fringe shift values are further analysed using an IBM Personal Computer.

A schematic diagram showing the various components mentioned above is given in figure 4 and a photograph of the experimental set up is shown in figure 5 . The components are described in detail in the following sections.

\subsection{The differential interferometer}

The differential interferometer used in the present experimental studies was supplied by Spindler and Hoyer, Göttingen, Germany. The basic parts of the differential interferometer are the light source, the interferometer head and the concave mirror. The working principle of the interferometer and its use in the measurement of heat transfer coefficient have been discussed in $\S 6$. In the instrument used in the present study, the light source is a high-pressure mercury vapour lamp in association with a heat filter and a colour filter for obtaining a monochromatic beam. Various colours namely blue, green, violet and yellow can be selected using the colour filters but yellow light is used in the present experiments because this is found to give pictures with the best contrast on exposure with black and white film. The interferometer mirror is a concave mirror of diameter $15 \mathrm{~cm}$ and radius of curvature $2.0 \mathrm{~m}$. The interferometer is set up on a triangular optical bench with standard profile DN 58110 . Its optic axis is kept $23.5 \mathrm{~cm}$ above the table. The light source is also placed

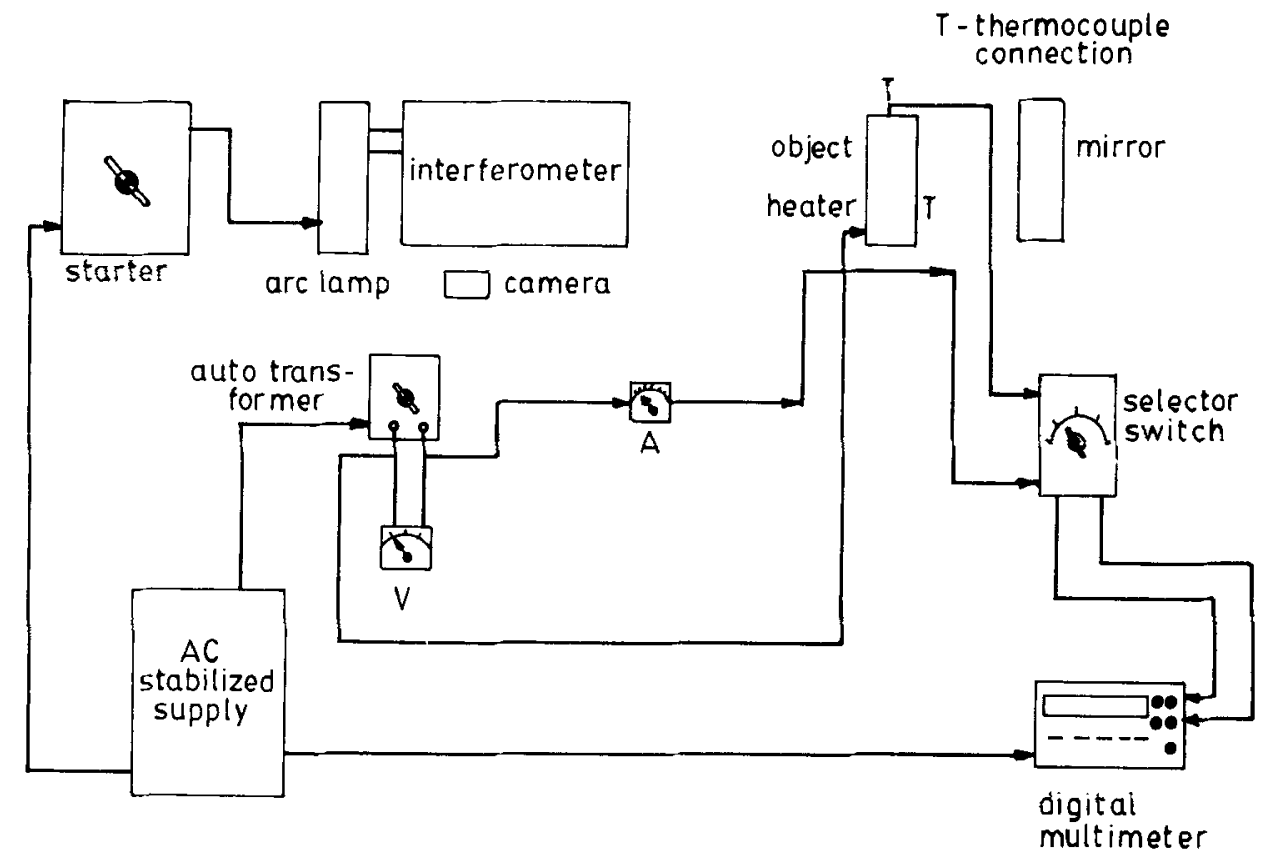

Figure 4. Schematic diagram showing the arrangement of the various components of the experimental set-up. 

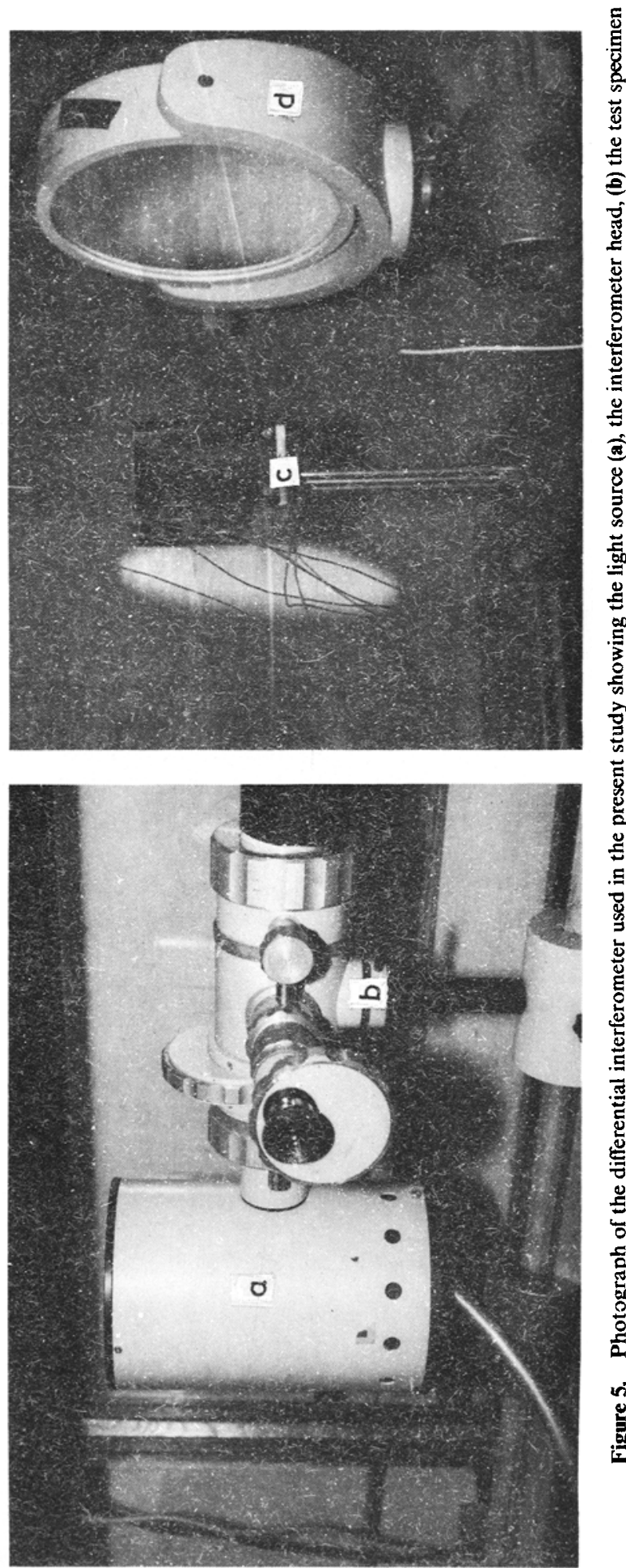

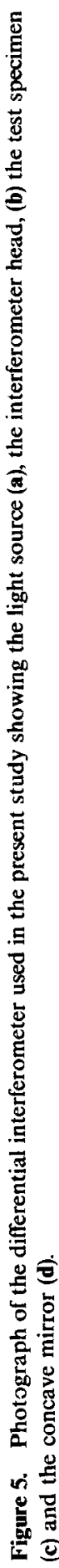


on the same bench and hence the mirror and the source can be easily moved to make proper adjustments. The test assembly is kept as close to the mirror as possible (the distance from the mirror being $0.34 \mathrm{~m}$ in the present case) so that the available field of view is effectively utilized. The distance between the test assembly and the differential interferometer head is therefore maintained at $1.66 \mathrm{~m}$.

There are three sensitivity positions for the interferometer head which can be obtained by selecting various Wollaston prisms in the main light path $\left(W_{1}\right.$ in figure 1$)$. Also three inter-fringe spacings (close, medium and wide) can be obtained by selecting various prisms in the eye-piece section $\left(W_{2}\right.$ in figure 1$)$. In the present experiments, the fringe spacings are always kept 'close' so that there are adequate fringes along the length of the analysed surface (the inter-fringe spacing is $3 \mathrm{~mm}$ and there are 23 fringes in a $70 \mathrm{~mm}$ length of the fin in the experiments). While analysing cases with low heat inputs to the system, where the fringe shifts are small, high and medium, sensitivity prisms are used as $W_{1}$, and in cases with higher values of heat input, the low sensitivity prism is used so that photographing can be effectively done without disturbances. The wedge angles and the corresponding sensitivities for the prisms $\left(W_{1}\right)$ are given below:

\begin{tabular}{lcc}
\hline No & Sensitivity & $\begin{array}{c}\text { Wedge angle } \\
\text { (degrees) }\end{array}$ \\
\hline 1 & Low & 1 \\
2 & Medium & 3 \\
3 & High & 5 \\
\hline
\end{tabular}

The differential interferometer and the test section are completely enclosed in a glass casing for maintaining a disturbance-free atmosphere. The whole system is assembled on a heavy wooden table and vibration-free test conditions are maintained.

\subsection{The test specimen}

Experiments have been carried out on a vertical isothermal flat plate, a single fin attached to a heated horizontal base and arrays of three fins on a heated horizontal base. The experiments were done with various heat input values, three fin materials and with different inter-fin spacings in the case of the fin arrays. Details of the experimental specimen used in the various cases are described more fully elsewhere (Sobhan et al 1990). A schematic of the fin-base assembly in the case of a fin array attached to a horizontal base is shown in figure 6.

\subsection{The power input system}

The power for heating the test specimen is input from the servo motor stabilized a.c. mains using a variable transformer and an ammeter-voltmeter combination for measuring the input power value. Nichrome wire heater used for heating the fin-base assembly consists of nichrome wire wound uniformly round a thin mica sheet so that the heating of the base is uniform. The heater is electrically insulated from the remaining portion of the assembly by sandwiching it between two thin mica sheets. 


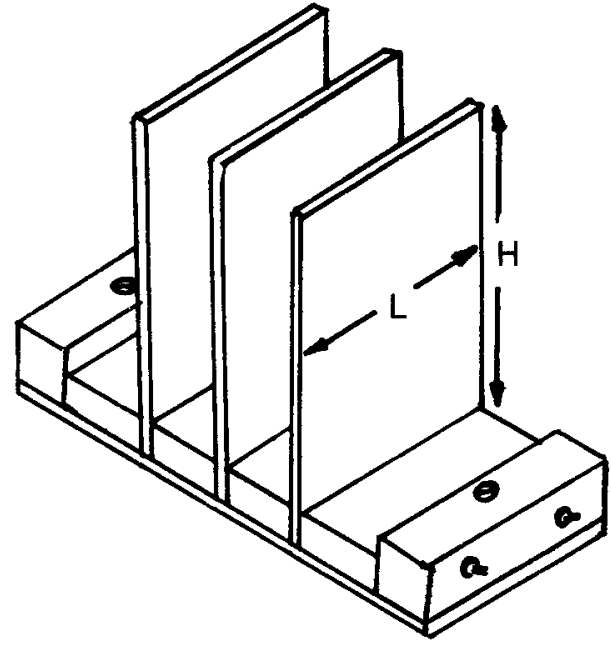

Figure 6. Schematic of the fin-base assembly in the case of a three-fin array.

In the case of the vertical flat plate the heater used was with variable spacing for the heater wire to achieve isothermal conditions.

\subsection{The temperature measurement system}

Copper constantan thermocouples made from wires of $0.2 \mathrm{~mm}$ diameter are used for the measurement of the temperatures. The thermo EMFs are measured using a digital voltmeter. A mercury in glass thermometer of $0 \cdot 1^{\circ} \mathrm{C}$ resolution is used for measuring the ambient temperature inside the test section, which serves as the reference temperature for the measurement.

\subsection{The photographic camera}

The fringe patterns are photographed, through the eye piece of the interferometer using an Asahi Pentax $35 \mathrm{~mm}$ photographic camera fitted with a macro-lens (focal length $15 \mathrm{~cm}$ ) of our design, on 80 ASA black and white film. These photographs are developed and magnified using a slide projector, from which the fringe shifts are measured.

\section{Adjustments of the interferometer set-up}

The various parts of the experimental set-up, namely the interferometer head, the test section and the mirror, have to be aligned properly for getting a high contrast fringe pattern. The distance from the mirror to the centre of the test section is measured accurately since this enters directly into the calculation of heat flux values. The alignment is perfect when the field of view through the eye piece is completely bright. It is ascertained that the fin base is perfectly horizontal and that the fin flats are perpendicular to the base. Also, the fin assembly is kept such that the fin flats are normal to the path of the light. This can be achieved by viewing through the eye piece and turning the fin assembly about a vertical axis such that the thinnest image of the fin edge is obtained in the field of view. The sliding door of the glass panel at 


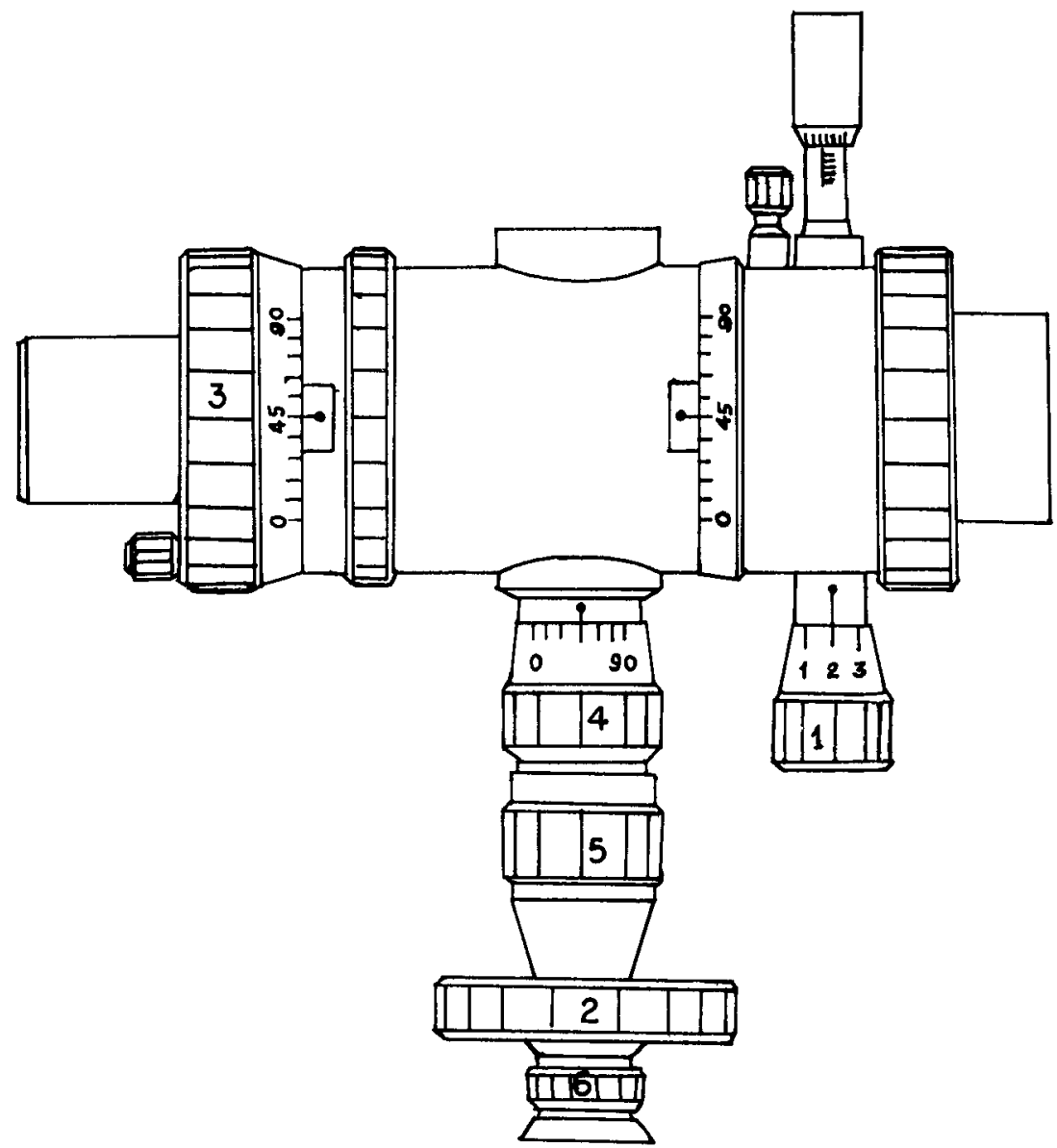

Figure 7. External view of the differential interferometer head showing the various controls for adjusting the instrument. 1 - Selector for Wollaston prism $W_{1}$ for obtaining different sensitivities of the instrument; 2 - selector for Wollaston prism $\mathrm{W}_{2}$ for obtaining various inter-fringe spacings; 3 - milled ring for turning the polariser; 4 -milled ring for turning the analyser; 5 -milled ring for focussing the image of the test specimen; 6 -milled ring for focussing the interference fringes by moving the analyser.

the test section is closed so that this section is completely free of any external disturbance. The required sensitivity of the differential interferometer can be selected by turning the milled ring, 1 , in figure 7 , which chooses prisms with different wedge angles as the first Wollaston prism $\left(\mathrm{W}_{1}\right.$ in figure 1$)$ and thus provides various angular separations between the coherent rays of light emerging from this prism. Milled ring, 2, is used for selecting suitable inter-fringe spacings, by choosing the second Wollaston prism ( $W_{2}$ in figure 1). By turning the milled rings, 3 and 4, through the same angles, the orientation of the interference fringes can be changed. For the present studies involving heat transfer from vertical surfaces, the interference fringes are kept horizontal. With the lamp on, the image is viewed through the camera placed at the eye piece and focused well. For this the milled ring, 5 , in figure 7 is used. The fringes are focused by moving the analyser, which is achieved by turning the milled ring, 6 . The lamp can be turned off after this and it is turned on only while photographing the fringe pattern. The sensitivity position is selected according to the requirement 
in the experiment. The fringe spacing is normally kept at the 'close' condition in the present experiments. The experimental procedure involves the following: (1) adjusting the interferometer, (2) heating the test specimen, (3) noting the appropriate temperatures, and (4) photographing the fringe patterns at the required instants. A detailed description of the experiments conducted are to be found in Sobhan (1989) and Sobhan et al $(1989,1990)$. In the following a few typical results are discussed.

\section{Analysis of the interferograms *}

The measurement procedure starts with developing the photographic plates, and projecting them on a screen under a known magnification, tracing the fringe pattern and measuring the local fringe shift values from the tracings. An alternate method is to use a digitizer to directly read the fringe shifts using a Personal Computer. A typical interferogram as viewed through the eye piece of the differential interferometer is shown in figure 8 for a fin array heated from a horizontal base. The measured interference fringe shift values and the tip and the base temperatures noted during the experimental runs go into the calculation procedure discussed in detail in Sobhan et al (1990) for obtaining the various heat transfer quantities.

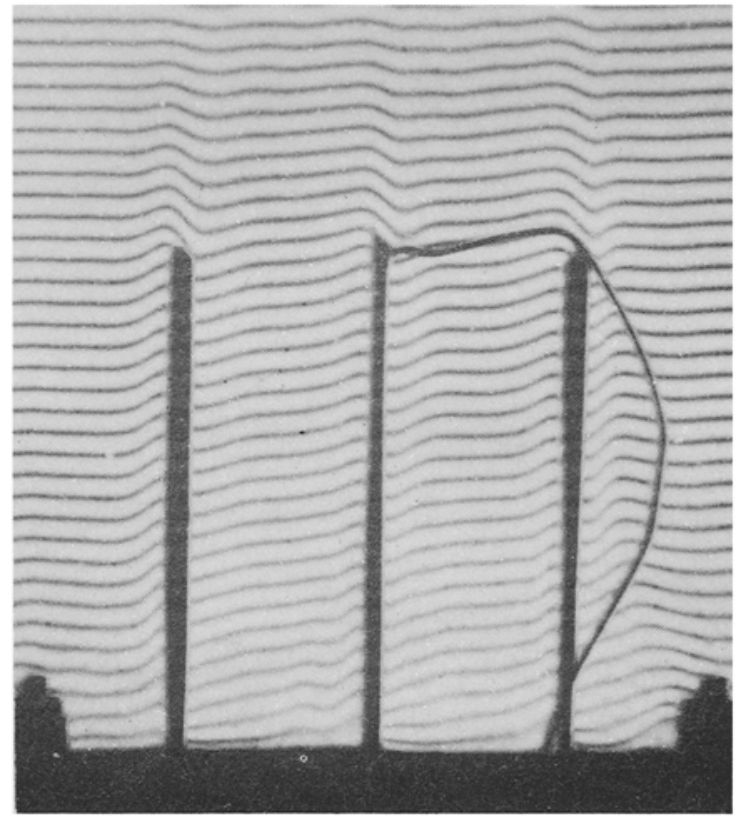

Figure 8. A typical fringe shift pattern in the case of a heated fin array. The fringes would have been horizontal if the system were isothermal.

\section{Results and discussion}

A large number of experiments were conducted in this laboratory for various applications like (1) isothermal vertical flat plate, (2) single fin with three materials (steady and unsteady state), and (3) three-fin array with three materials (steady and unsteady state with three different fin spacings). The three materials considered are aluminum, brass and mild steel. Typical results are discussed in the following. Details may be found in Rammohan Rao \& Venkateshan (1990) and Sobhan et al (1989, 1990). 


\subsection{Vertical isothermal flat plate}

The flat plate is fabricated by sandwiching a flat element heater in between two uniform aluminum plates of dimensions $100 \mathrm{~mm} \times 60 \mathrm{~mm} \times 1.5 \mathrm{~mm}$. The heater consists of nichrome wire wound round mica sheets, insulated electrically on both sides. A low input power value of $5 \mathrm{~W}$ is applied to the heater. At this heating level, the convective pattern is laminar $\mathrm{Gr}_{x}=1.96 \times 10^{6}$, which is calculated from the measured temperatures. Three thermocouples attached to one of the surfaces along a diagonal read $54.4 \pm 0.6^{\circ} \mathrm{C}$. The local Nusselt numbers are calculated by analysing

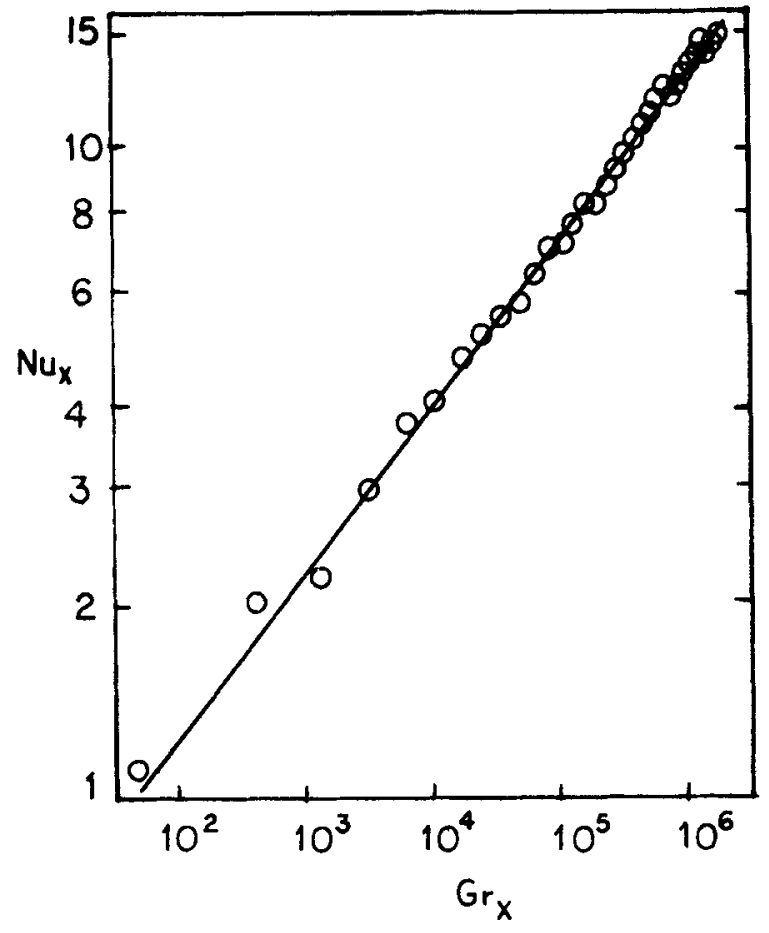

Figure 9. Comparison of the steady state experimental results of a vertical isothermal flat plate with integral solution (Chapman 1984) (O experimental points: - theoretical formula).

the interferograms and compared with the integral solution (Chapman 1984) for the local Nusselt number in a vertical isothermal flat plate, given by

$$
\mathrm{Nu}_{x}=0.508 \mathrm{Gr}^{0.25} \operatorname{Pr}^{0.5} /(0.952+\operatorname{Pr})^{0.25}
$$

The r.m.s. values of the deviation between experimental values and those obtained from the above expression are found to be 0.261 for the Nusselt number and 0.287 for the heat transfer coefficient. As a percentage of the average heat transfer coefficient, the deviation is within $\pm 5.5 \%$. Figure 9 shows that the experimental and the theoretical values are in excellent agreement.

The temperature profiles are obtained at various locations from the tip using the method described in $\$ 6.4$. The results are compared with Ostrach's similarity solution in figure 10. It is seen that the results fall within a narrow band which also encloses the Ostrach's solution. The isotherms are plotted in figure 11 from these temperature profiles. Figure 12 shows the expected interference (isotherm) pattern (notice the poor resolution) if the same experiment were to be performed with an MZI. 


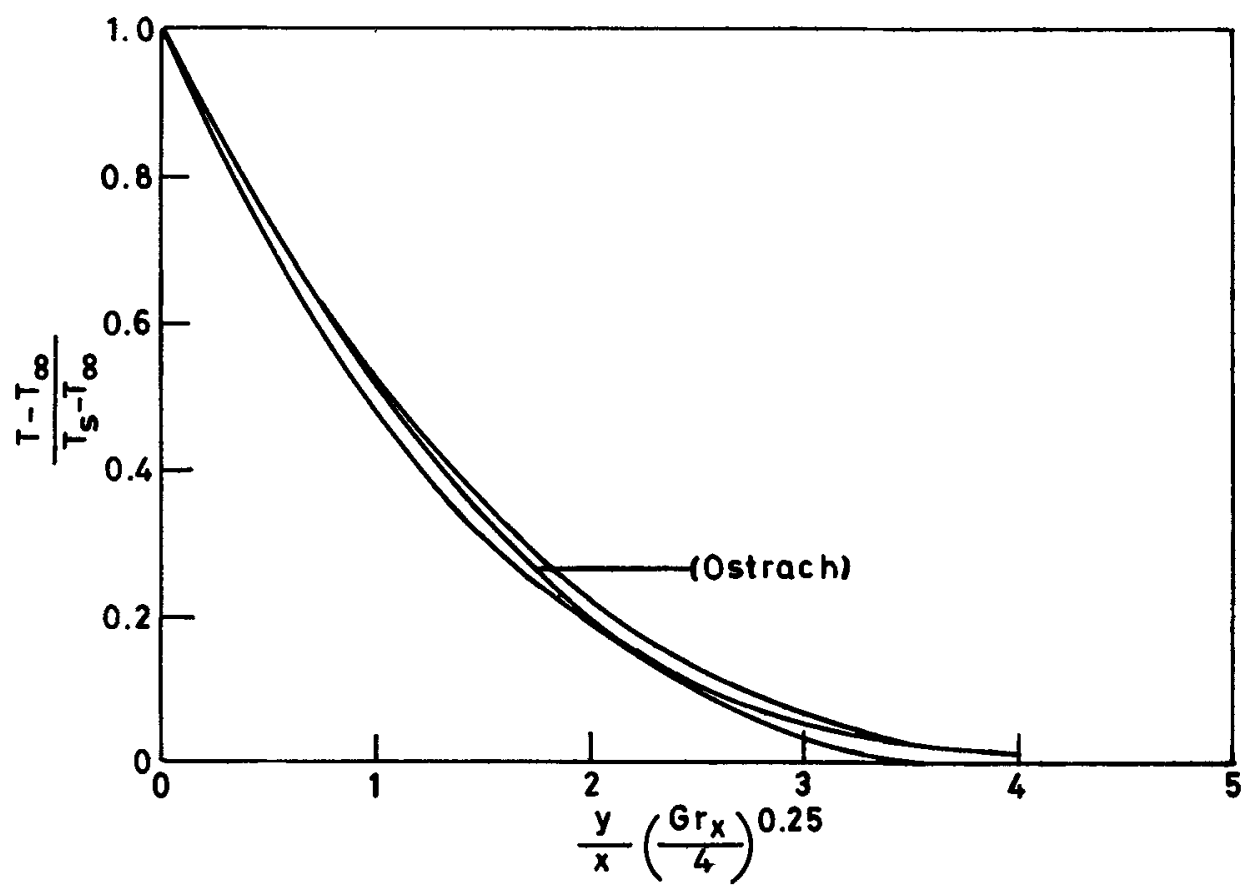

Figure 10. Temperature profiles in the case of an isothermal flat plate for various $\mathrm{Gr}_{x}$. The temperature values are bracketed for the full curves for $10^{3} \leqslant \mathrm{Gr} \leqslant 10^{6}$.

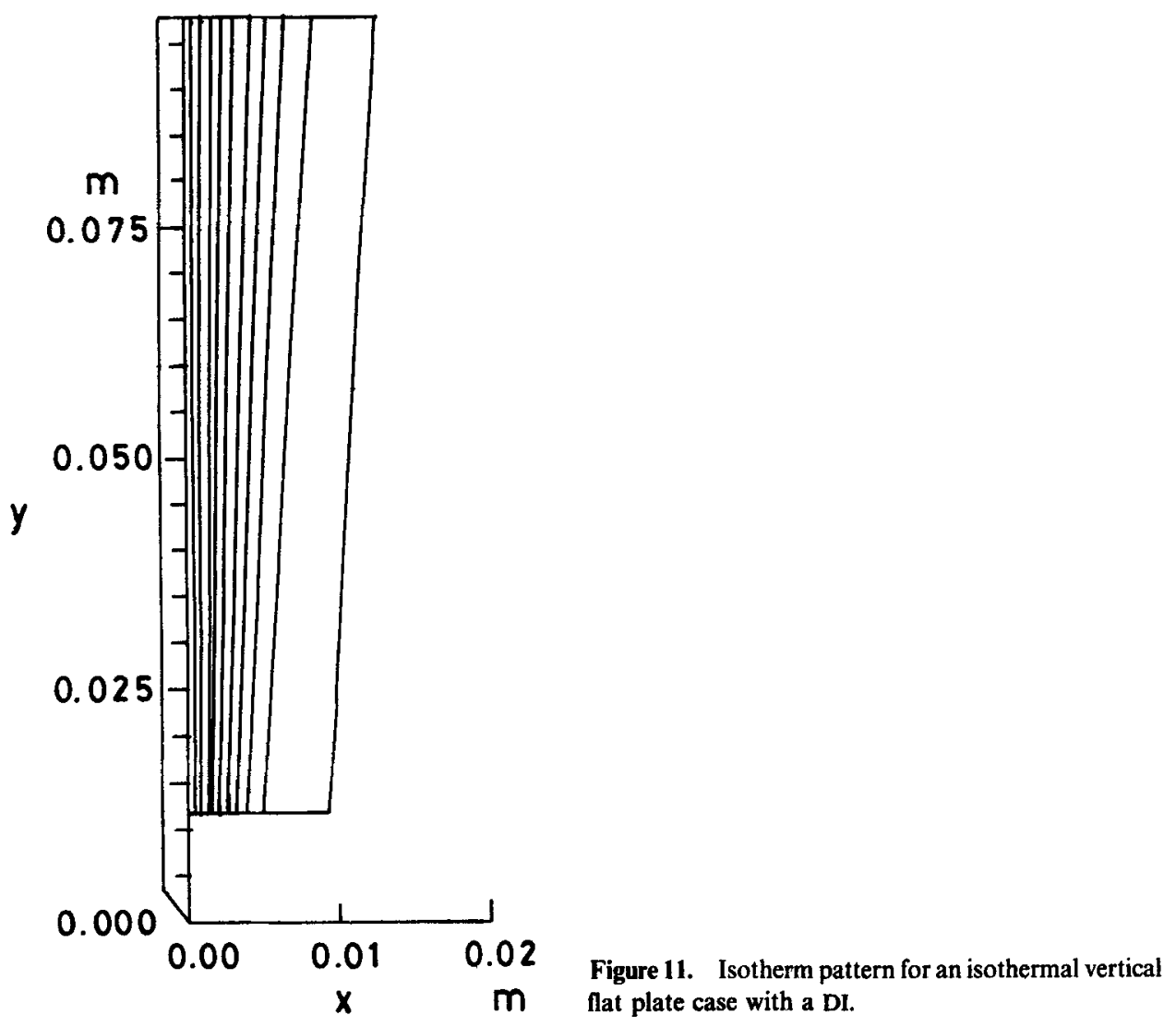




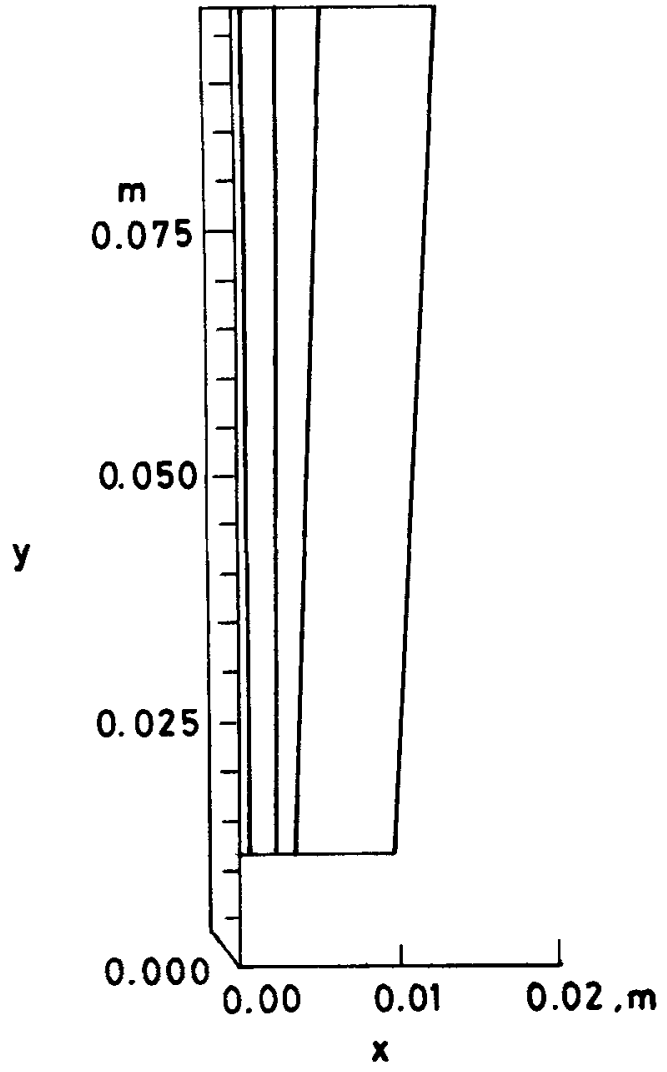

Figure 12. Expected isotherm pattern for the earlier case (fig. 11) with an MZI.

\subsection{Single fin}

The fins are made of polished metal plates (for reducing the radiation effect) of dimensions $80 \mathrm{~mm} \times 50 \mathrm{~mm}$ for the flats. The thickness of the plate $(1 \mathrm{~mm})$ is very small comapared to the other dimensions, and it can be assumed that the heat transfer within the fin is one dimensional. In the assembly (schematic is shown in figure 13), the fin is kept vertical over the base plate with the help of the mild steel blocks, the end-blocks and the through-bolts. The end blocks are of a bigger size than the other blocks to ensure that there is no warping of the fin base. The height of the fin exposed outside the mild steel blocks for heat transfer is $70 \mathrm{~mm}$. The flat element heater is

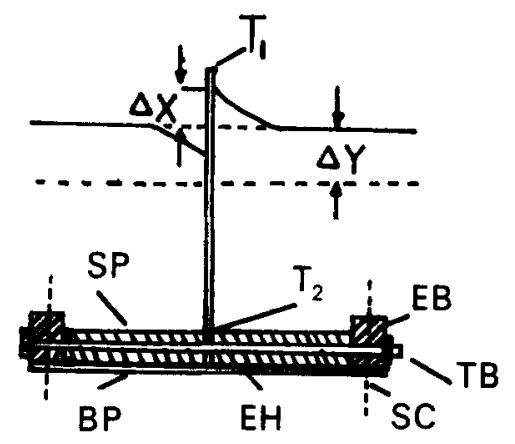

Figure 13. Cross-sectional view of the fin-base assembly in the case of a single fin attached to a heated horizontal base. $\mathrm{SP}$-spacer block; EH - electric heater; BP-base-plate; TB through-bolt; EB-end-block; SC-screw; T-thermocouple. 


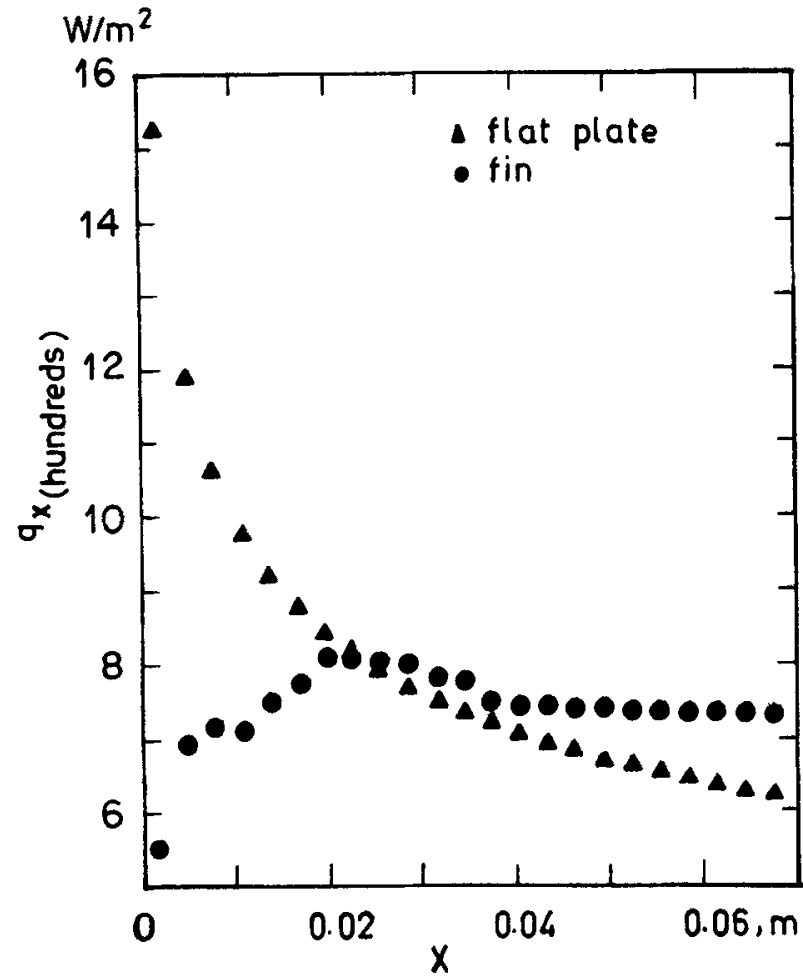

Figure 14. Comparison of the local heat flux values in the case of a single aluminum fin in the steady state with a heater input of $38.4 \mathrm{~W}$, with an isothermal flat plate.

placed between the mild steel blocks and the back plate. The heater is uniformly wound so that the whole base is heated uniformly. The assembly is kept over a mild steel stand. Two copper-constantan thermocouples are attached to the fin at the positions shown in the figure, and connected to the temperature measurement system. Figure 14 shows a typical plot for variation of the local heat flux along the height of the fin. Also plotted is the variation of heat flux in the case of an isothermal flat plate. It can be seen that the heat flux in the case of a single fin starts with a small value at the base, attains a peak value at about a quarter height and then falls steadily to a uniform value. A similar trend was observed for all the cases with various materials and heating levels. The development of the temperature profiles is plotted in figure 15 in terms of the similarity variables so as to compare the data with those obtained for an isothermal flat plate.

\subsection{Array of fins}

The fin assembly consists of three polished metal plates placed vertically over a horizontal base and separated from each other using mild steel spacers whose width can be chosen to give the required inter-fin spacing (schematic is shown in figure 6). The base is heated uniformly using a flat heater placed below the spacer blocks. The central fin has two thermocouples attached to it at the tip and the base respectively. The dimensions of the fins after assembly are the same as for the single fin considered above.

Figure 16 shows the variation of the local heat flux along the height of the middle fin compared to that of a single fin. This is a typical case and a similar trend is 


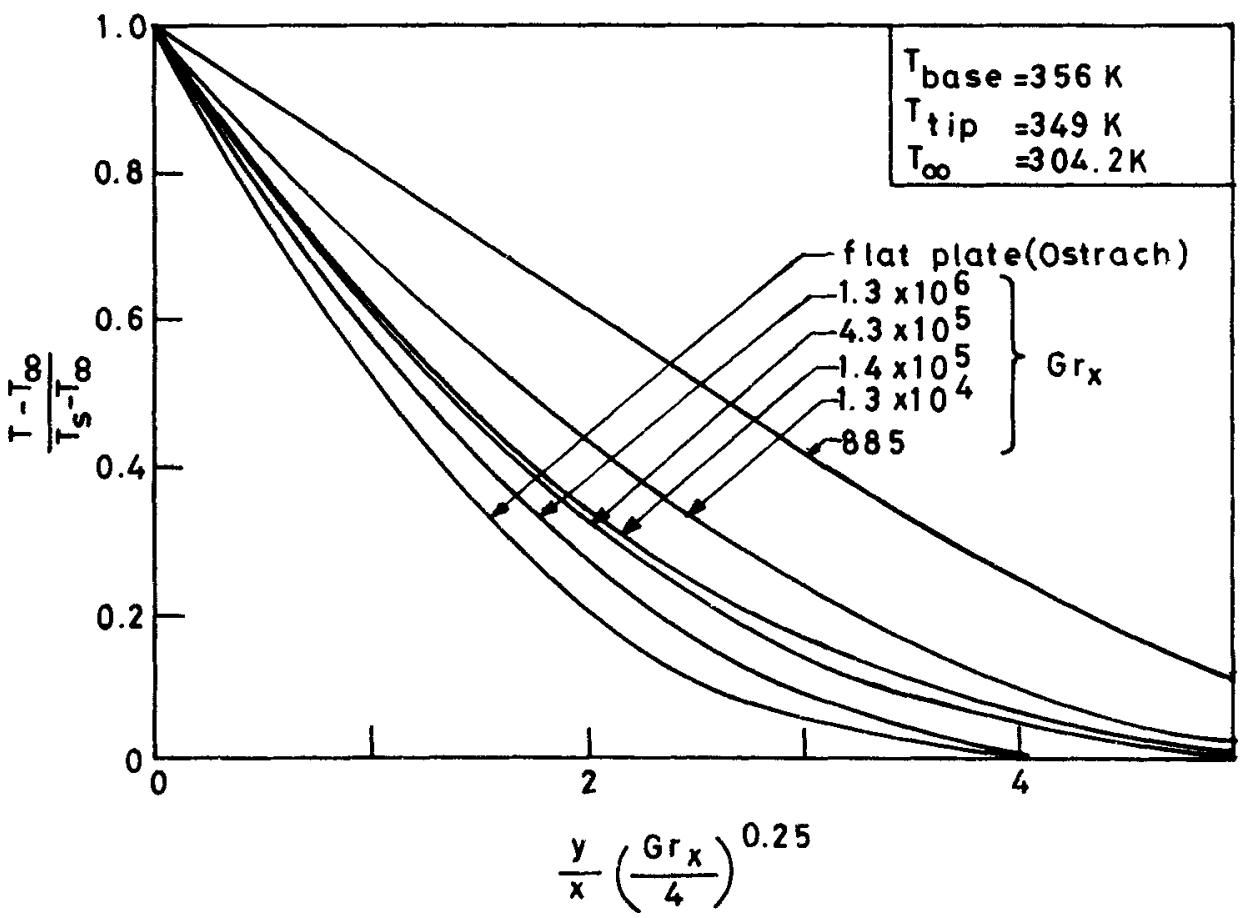

Figure 15. Temperature profile development from the base to tip in the case of a singie fin.

observed in all the cases with different materials and with different fin spacings. In contrast to the case with a single fin, in the case of a fin array, the heat flux shoots up at the tip. The reason for this variation is attributed to the chimney flow which is caused by the flow of air from the sides. For a detailed analysis of temperature profile estimation, the fin assembly used was different from that used for heat transfer calculations. This fin array consisted of four equispaced fins without the base extension.

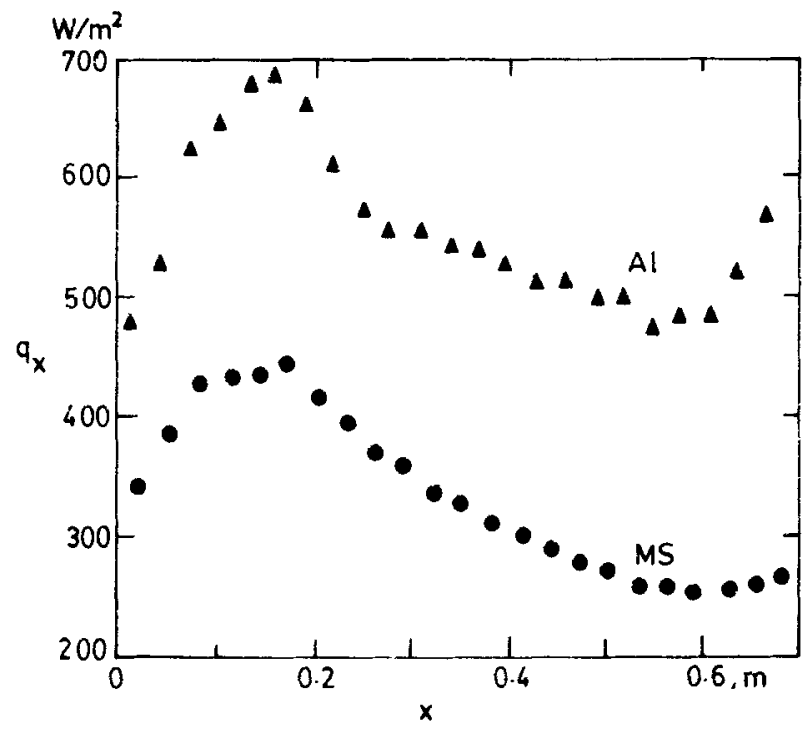

Figure 16. Variation of the local heat fiux for aluminum and mild steel fins in an array of fins, with $10 \mathrm{~mm}$ spacing and $38.4 \mathrm{~W}$ heater input, at the steady state. 


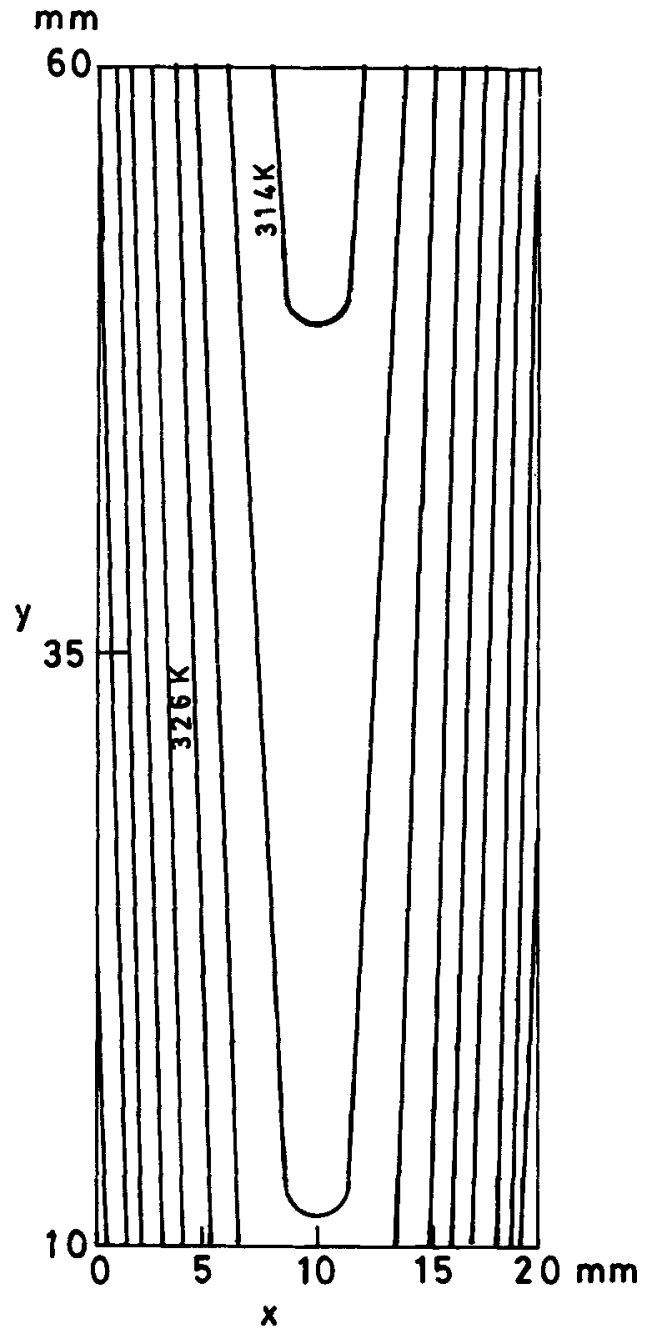

Figure 17. Isotherms in the case of an array of four fins with $20 \mathrm{~mm}$ fin spacing (plot depicts the temperature pattern in the space between the two middle fins).

The temperatures are estimated in the space between the two middle fins. Temperature profiles are obtained at seven locations from the base. Using this data an isotherm plot is made as shown in figure 17.

\section{Concluding remarks}

The theory and constructional details of a differential interferometer have been described in detail. Comparisons between a DI and a Mach-Zehnder interferometer have been made. Sample calculation shows that, if the same size of the test set-up is analysed with an MZI the number of interference lines would be very small, whereas in the case of a DI the number of fringe lines is independent of the object size. This has been the reason for having larger object lengths analysed wherever an MZI has been used in free convection experiments performed in air. For example in Hauf \& Grigull (1970), the object length (along the light rays) used is $500 \mathrm{~mm}$ in air and $50 \mathrm{~mm}$ in water. It is not possible to use an MZI where the optical path is not enough to get 
a minimum number of interference lines and where one does not have any control over it. Demonstration of the temperature estimation clearly reveals that the DI can also be used to obtain good temperature estimates.

\section{List of symbols}

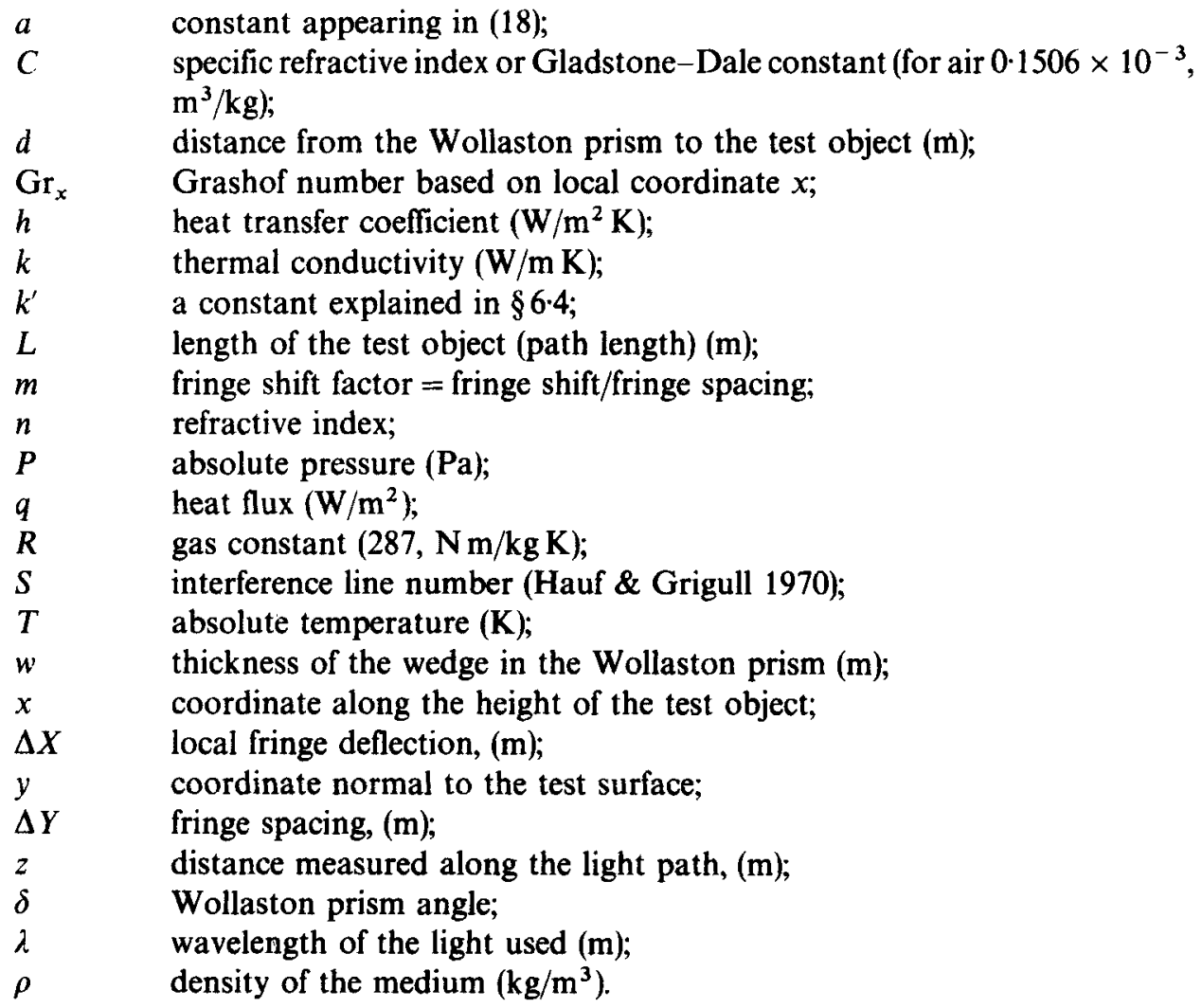

\section{Subscripts}

$\begin{array}{ll}w & \text { referred to the wall conditions; } \\ \infty & \text { referred to the ambient conditions; } \\ o & \text { ordinary ray; } \\ e & \text { extraordinary ray. }\end{array}$

\section{References}

Black W Z, Carr W W 1971 Application of differential interferometer to the measurement of heat transfer coefficients. Rev. Sci. Instrum. 42: 337-340

Black W Z, Norris J K 1974 Interferometric measurement of fully turbulent free convective heat transfer coefficient. Rev. Sci. Instrum. 45: 216-218

Chapman A J 1984 Heat transfer (4th edn.) (London: Macmillan)

Dosanjh D S (ed.) 1971 Modern optical methods in gas dynamic research (New York: Plenum)

Dyson J 1970 Interferometry as a measuring tool (London: The Machinery Publication Co.)

Edser E 1949 Light for students (London: Macmillan) 
Hauf W, Grigull U 1970 Optical methods in heat transfer. Adv. Heat Transfer 6: 134-365

Rammohan Rao V, Venkateshan S P 1990 Natural convection heat transfer and associated temperature fields in fins and horizontal fin arrays, to be presented at the 3rd ASME-JSME Thermal Engineering Joint Conference, 1991

Schrader W 1978 Manual on differential interferometer (Braunschweig: Spindler \& Hoyer)

Smith F G, Thomson J H 1977 Optics (London: ELBS/John Wiley)

Sobhan C B 1989 Free convection studies on horizontal fins and fin arrays via differential interferometry, Doctoral thesis, Indian Institute of Technology, Madras

Sobhan C B, Venkateshan S P, Seetharamu K N 1989 Experimental analysis of unsteady free convection heat transfer from horizontal fin arrays. Warme und Stoffubertragung 24: 155-160

Sobhan C B, Venkateshan S P, Seetharamu K N 1990 Experimental studies on steady free convection heat transfer from fins and fin arrays. Warme und Stoffubertragung 25: (in press)

Starling S G, Woodall A J 1963 Physics (London: ELBS/Longman Green) 